\title{
Performance Analysis of Multi-Carrier Two-Way Cooperative Communication with Relay Selection Diversity
}

\author{
Muhammad Abrar, Xiang Gui, and Amal Punchihewa
}

\begin{abstract}
Cooperative communication provides most of the advantages as multi-antenna systems due to availability of independent propagation paths between mobile terminals and the base station. In this paper we investigate the performance of Multi-carrier Two-way Two-hop (TW-TH) cooperative communication network in the presence of Rayleigh fading channel. We analyze a simple relay selection diversity scheme in TW-TH cooperative network. The two power allocation schemes with relay selection are discussed. Performance analysis shows that relay selection diversity scheme provides better performance than fixed relaying regardless of the availability of direct path between users.
\end{abstract}

Index Terms-Cooperative communication, cooperative diversity, relay selection, two-way relaying

\section{INTRODUCTION}

Cooperative communication has attracted attention of large number of researchers around the world due to the increased reliability of received signal as well as the extended coverage area [1]-[4]. The concept of relaying was first introduced by Van der Meulen [5] and Cover and EI Gamal [6]. This concept of using relaying was different from the recent concept of relaying being used in cooperative communication The authors in [5] and [6] used only additive white Gaussian noise (AWGN) channel and there is no concept of diversity at that time. Thus the main reason of using relay was to provide help to the main channel. In cooperative communication, the relays are being used to achieve diversity in fading wireless channels besides enhancing the coverage area. In cooperative communication, users co-operate themselves to transfer information from one user/source to other user/destination [7] Relay can be of fixed or moving like mobile devices. In traditional half duplex one-way relaying, four time slots are required to complete the exchange of information between two users, hence resulting in the loss of system spectral efficiency. To overcome this loss, two-way/bidirectional relaying was proposed in [8].

\section{A. Cooperative Protocols}

There are two common relaying protocols proposed in literature namely Amplify and Forward (AF) and Decode \&

Forward (DF). The AF is a simple protocol in which relay station forward the scaled version of received noisy signal from one user to another user. Besides simplicity and low cost, noise amplification at relay is a major drawback. In DF, relay first detects the original signal bits, re-encode them and then

Manuscript received November 13, 2012; revised April 1, 2013.

The authors are with the School of Engineering and Advanced Technolog, Massey University, New Zealand (e-mail: M.Abrar@massey.ac.nz). forward to destination. There is a large number of other protocols proposed in literature, but all of these are created from these two basic protocols [7].

\section{B. Multi-Carrier System}

Orthogonal Frequency Division Multiplexing (OFDM) is a dominant technology for wireless broadband communications and has already been employed in different communication standards. OFDM provides simple equalization solution at receiver by transforming frequency selective channels into frequency-flat fading channels [9]. OFDM has already been employed in a number of communication standards including Digital Audio Broadcasting (DAB), Wireless LAN standards and IEEE 802.16 Broadband Wireless Access System . OFDM is also the chosen modulation technique in next generation wireless standards such as IEEE 802.16j, IEEE. $802.16 \mathrm{~m}$ and 3GPP LTE-A where relaying systems are also being incorporated. The combination of cooperative relaying with multicarrier system provides promising design for next generation of wireless networks.

The purpose of this work is to better understand the two-way cooperative relaying in multicarrier scenario with selection of best relay. In this paper we investigate the performance of Multi-carrier Two-way Two-hop (TW-TH) cooperative communication network in the presence of Rayleigh fading channel.

\section{Selective Relaying}

Selection of suitable relay is a challenging issue in cooperative communication systems. Some efforts have been already made in analyzing the impact of selection of best relay in multi relay networks but most of the work is related to traditional one-way relaying. The relay selection problem for two-way relaying however is not much studied. In [10] relay selection is investigated using cross layer design for two-way relaying. In [11] a relay selection scheme for two-way relaying is proposed to maximize sum-rate capacity with decode and forward protocols. In this paper, we propose two-way cooperative communication systems with the amplify-and-forward protocol over a multiple distributed relay network where only one best relay among many available relays is selected according to an average of link channel conditions. We analyze the impact of selection relay in terms of Symbol Error Rate (SER) performance.

\section{Outline}

The remaining paper is organized as follows. The system model is formulated in Section-II, while relay selection and power allocation is described in Section-III. In Section-IV we provide performance analysis with simulation results. Finally, Section-V concludes our results. 


\section{SYSTEM DESCRIPTION AND NETWORK MODEL}

We consider a mobile cooperative network in which mobile station (MS) and base station (BS) want to exchange information. We consider two separate models. In the first model, we consider that there is no direct link between the MS and $\mathrm{BS}$, and the exchange of information is via relay link only. In the second model, we assume that a direct link is also established along with relay link. We consider that there are $R$ relays that can provide cooperation but in this paper only one best relay is selected for each multi-carrier symbol. A selection criterion is explained in section-III. Time Division Duplex (TDD) is used to achieve separation between uplink and downlink transmission to and from relay respectively as all relays are working in half duplex mode. All the channels are modeled as Rayleigh flat fading, with unit variance and additive white Gaussian noise (AWGN) with unit power spectral density. Both users and relays are equipped with single antenna. We consider that all channels remain constant for two time slots. We also assume that channel state information (CSI) is known to all nodes.

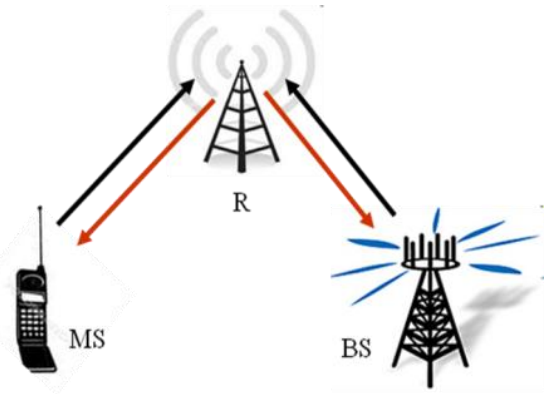

(a)

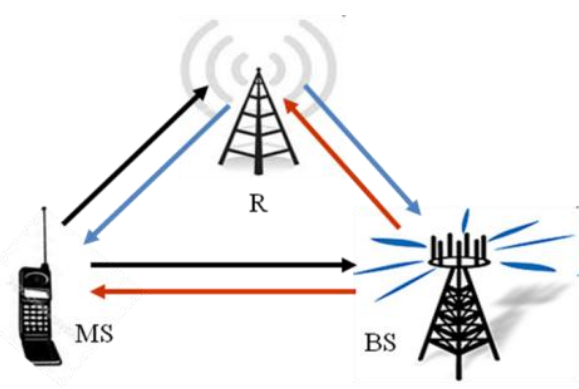

(b)

Fig. 1. Two-way cooperative communication: (a) without a direct link, (b) with a direct link

\section{A. System Model-1}

In the first cooperative system model as shown in Fig. 1(a), we assume that there is no direct link and all information between MS and BS is exchanged via a single best relay. Two time slots are required to complete the transfer of information between two users. During the first time slot both MS and BS transmit their signal to the relays. All relays receive the combined signal of MS and BS due to broadcast nature of wireless channel. But only one selected best relay amplifies this combined signal and then transmits to both MS and BS in the second time slot. Considering that $S_{1}$ and $S_{2}$ are the transmitted OFDM symbols of the MS and BS, respectively. The combined signal received at the relay can be expressed as (1):

$$
Y_{r} \sqrt{P_{1}} S_{1} h_{m r}+\sqrt{P_{2}} S_{2} h_{b r}+n_{r}
$$

where $Y_{r}$ is the combined received signal and $h_{m r}, h_{b r}$ are the channel gains of the MS-Relay link and the BS-Relay link, respectively. The term $n_{r}$ represents the AWGN at the relay while $P_{1}$ and $P_{2}$ are the transmission powers of the MS and the $\mathrm{BS}$, respectively. The received signal $Y_{r}$ is amplified by the relay with scaling factor $\beta_{r}$ as proposed in [4]:

$$
\beta_{r}=\sqrt{\frac{P_{r}}{1+\left|h_{m r}\right|^{2} P_{1}+\left|h_{b r}\right|^{2} P_{2}}}
$$

where $P_{r}$ is the transmission power of the relay. Both the MS and BS receive the amplified signals, $Y_{m}$ and $Y_{b}$ from the relay in the second time slot, respectively. By using (1) and (2) the received signals at $\mathrm{MS}$ and $\mathrm{BS}$ can be represented as, respectively:

$$
\begin{gathered}
Y_{m}=\beta_{r} \sqrt{P_{1}} S_{1} h_{m r} h_{r m}+\beta_{r} \sqrt{P_{2}} S_{2} h_{b r} h_{r m}+N_{m} \\
Y_{b}=\beta_{r} \sqrt{P_{1}} S_{1} h_{m r} h_{r b}+\beta_{r} \sqrt{P_{2}} S_{2} h_{b r} h_{r b}+N_{b}
\end{gathered}
$$

The terms $N_{m}$ and $N_{b}$ in (3) and (4) represent the noise term which contains both the amplified version of AWGN components received from the relay and the AWGN component at the receiving node itself, respectively.

\section{B. System Model-2}

In the second cooperative system model as shown in Fig.1 (b), we consider that a direct path is also present between MS and BS along with the relaying path. In this communication there are three time slots required to complete the transfer of information between MS and BS. Equations (1) and (2) of previous model stand the same for this model but there is an addition of signals received from the direct link in (3) and (4). After addition of direct transmission to the received signals at both MS and BS can be represented by (5) and (6), respectively:

$$
\begin{aligned}
& Y_{m}=\beta_{r} \sqrt{P_{1}} S_{1} h_{m r} h_{r m}+\beta_{r} \sqrt{P_{2}} S_{2} h_{b r} h_{r m}+\sqrt{P_{2}} S_{2} h_{b m}+N_{m} \\
& Y_{b}=\beta_{r} \sqrt{P_{1}} S_{1} h_{m r} h_{r b}+\beta_{r} \sqrt{P_{2}} S_{2} h_{b r} h_{r b}+\sqrt{P_{1}} S_{1} h_{m b}+N_{b}
\end{aligned}
$$

The second last term in (5) and (6) shows the signal which is received from the direct path, $h_{b m}$ and $h_{m b}$ are respective channel gains in the direct link between MS and BS.

In both models, the received signal at both MS and BS also consists of their own transmitted signal known as selfinterference signal. With the knowledge of channel and its own signal at each user, this self-interference signal can be subtracted from the received signal. The self-interference can also be avoided by allocating different subcarriers to each user. We use $N$ subcarriers in both systems. We allocate half of the subcarrier to MS and the remaining half to $\mathrm{BS}$ as given in (7). 


$$
\begin{cases}M S:\left[1 \sim \frac{N}{2}\right] & \text { Subcarriers } \\ B S:\left[\frac{N}{2}+1 \sim N\right] & \text { Subcarriers }\end{cases}
$$

In this way at the relay there is no overlap between subcarriers and both MS and BS can get their required signal without any self-interference on allocated subcarriers.

\section{RELAY SELECTION AND POWER ALLOCATION}

\section{A. Relay Selection}

The optimal relay selection scheme in which one best relay is selected among available relays is analyzed in two way relaying. The selection of best relay is based on the average channel link condition between MS-relay and BS-relay. We assume that each subcarrier in one OFDM symbol examines same channel conditions and these remain same for two time slots, i.e., $h_{m r}=h_{r m} \& h_{b r}=h_{r b}$. The relay selection algorithm consists of two steps. In the first step the average channel gain for each relay is calculated by BS as it knows all channel conditions or can get by training methods.

$$
H_{n}=\left(\frac{\left|h_{m r}\right|^{2}+\left|h_{b r}\right|^{2}}{2}\right) \quad n=(1, \ldots M)
$$

In (8), $H$ shows the average channel gains for relays willing to cooperate and subscript $n$ denotes the relay index. After acquiring all average channel gains, in the second step, the best relay with maximum average channel gain is selected and power allocation is made in such a way that whole relaying power is allocated to that relay and all other relays are made unaffected by making their transmit power equal to zero. We name this process as relay selection diversity. This type of selection diversity is different from the traditional selective diversity which is performed at the receiver. In traditional selective diversity, all paths use some transmit power, while in our proposed selective diversity only selected path uses the whole power hence providing better performance. In cellular network, base station itself acts as the central /decision unit with knowledge of all channel link gains. In adhoc network a terminal acting as a server can also act as the central/decision unit.

\section{B. Power Allocation}

In most early publications, equal power allocation is assumed where all terminals transmit signal with the same power. In this scenario $P_{1}=P_{2}=P_{r}$ but this allocation is not fair for two-way cooperative communication protocol. In TW-TH cooperative protocol relay deals with signals of both users at the same time, so it needs more power as compared to one-way relaying where relay forwards signal of each user individually in different time slots. In this scenario, each user allocates half of the power to the relay, hence the allocation of $2 P_{I}=2 P_{2}=P_{r}$ can be described as equal power allocation in TW-TH cooperative communication protocol. In this paper we provide results with both power allocation schemes.

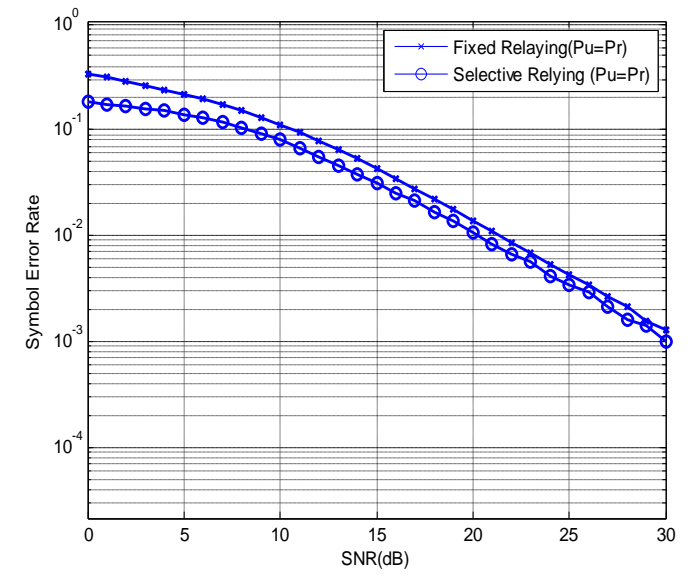

Fig. 2. SER performance with $P_{1}=P_{2}=P r$.

\section{PERFORMANCE ANALYSIS}

The performances of proposed schemes are evaluated by simulating the algorithm in MATLAB environment. Quadrature Phase Shift Keying (QPSK) modulation is employed in all simulations. A total of 106 symbols are used in each simulation. For TW-TH cooperative communication we use both equal power allocations within the constraint of the total transmitted power. The SER curve is defined as function of $P / N_{\mathrm{o}}$, the signal-to-noise-ratio, where $N_{\mathrm{o}}$ is the noise density and $P$ is the total transmitted power.

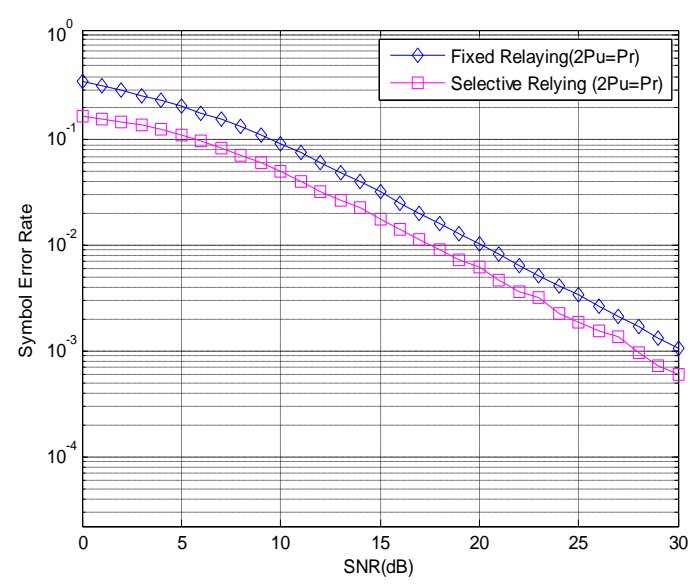

Fig. 3. SER performance with $2 P_{1}=2 P_{2}=P_{r}$

Fig. 2 and Fig. 3 show SER performance comparison of fixed relaying and our proposed selective relaying under unfair equal power allocation in which each terminal transmits signal with the same power and fair equal power allocation respectively as discussed in the previous section, respectively. It is also assumed that there is no direct link in both fixed and selective relaying cases and only relaying link is used to carry information. It is clear from these results that selective diversity is achieved and it outperforms fixed relaying under both power allocation schemes. Fig.4 shows the SER performance of selective relaying without direct path and fixed relaying with direct path under equal power allocation. The results show that our proposed selective relaying achieves the same performance as compared with fixed relaying with direct path with less time slots used hence increasing spectral efficiency. 


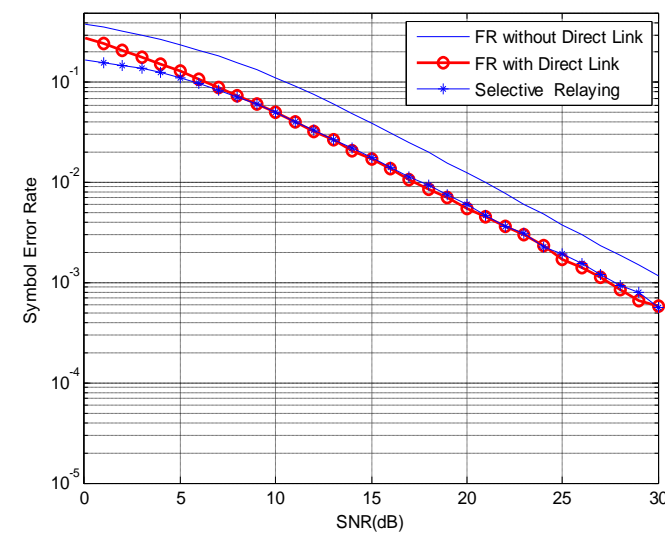

Fig. 4. SER performance comparison

\section{CONCLUSIONS}

In this paper, we analyzed the SER performance of multi-carrier TW-TH cooperative communication in fading channel under two equal power allocation schemes. A simple relay selection scheme is proposed to provide diversity when there is no direct link established between user and destination and only the best relay is used. Simulation results show that our proposed relay selective diversity scheme provides better performance than fixed relaying when no direct path is available. It achieves the same SER performance as two-way relaying in the presence of direct path but with higher spectral efficiency, because it needs only two time slots instead of three. The extensions of this work to multiuser, multi-relay network, and subcarrier allocation with optimization of power are our future work.

\section{REFERENCES}

[1] E. Erkip, A. Sendonaris, and B. Aazhang, "User cooperation diversity. part II. implementation aspects and performance analysis," IEEE Transactions on Communications, vol. 51, pp. 1939-1948, 2003.

[2] E. Erkip, A. Sendonaris, and B. Aazhang, "User cooperation diversity. part I. system description," IEEE Transactions on Communications, vol. 51, pp. 1927-1938, 2003.

[3] A. Nosratinia, T. Hunter, and A. Hedayat, "Cooperative communication in wireless networks," IEEE Communications Magazine, vol. 42, pp. 74-80, 2004.

[4] D. Tse, J. Laneman, and G. Wornell, "Cooperative diversity in wireless networks: Efficient protocols and outage behavior," IEEE Transactions on Information Theory, vol. 50, pp. 3062-3080, 2004.

[5] E.C. Meulen, "Three-terminal communication channels," Advances in Applied Probability, vol. 3, pp. 120-154, 1971.

[6] T. Cover and A. Gamal, "Capacity theorems for the relay channel," IEEE Transactions on Information Theory, vol. 25, pp. 572-584, 1979.

[7] M. Abrar, X. Gui, A. Punchihewa, S. Khan, and M. Iqbal, "Cooperative diversity versus antenna diversity in wireless communication systems," NISS-2010, pp. 23-26.

[8] B. Rankov and A. Wittneben, "Spectral efficient protocols for half-duplex fading relay channels," IEEE Journal on Selected Areas in Communications, vol. 25, pp. 379-389, 2007.

[9] X. Gui, "Iterative MIMO-OFDM detection under nonlinear channel distortions," ATNAC, pp. 1-6, 2009.
[10] L. Ding, M. Tao, F. Yang, and W. Zhang, "Joint scheduling and relay selection in one- and two-way relay networks with buffering," Communications Society, 2009.

[11] T. Oechtering and H. Boche, "Bidirectional regenerative half-duplex relaying using relay selection," IEEE Transactions on Wireless Communications, vol. 7, pp. 1879-1888, 2008.

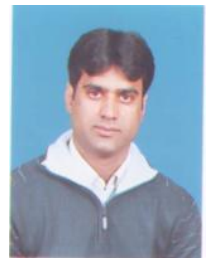

Muhammad Abrar received the B.Sc. degree in Electrical Engineering with First Class from the University of Engineering and Technology (UET) Taxila, Pakistan, in 2000 and the M.Sc. degree from UET Lahore, Pakistan in 2007. He is currently pursuing his $\mathrm{PhD}$ studies at the School of Engineering and Advanced Technology (SEAT), Massey University, New Zealand. His current research interests are wireless networks including MIMO, Relay networks and cooperative communications.

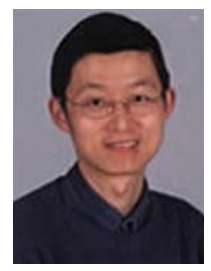

Xiang Gui received his B.S. and M.S. degrees from Shanghai Jiao Tong University, China, in 1991 and 1994, respectively, and the $\mathrm{PhD}$ degree from the University of Hong Kong in 1998, all in electrical engineering. In 1994, he was an Assistant Lecturer in the Department of Electrical Engineering, Shanghai Jiao Tong University, China. From 1998 to 2003, he worked at Nanyang Technological University, Singapore, first as a Research Fellow then as an Assistant Professor. In 2003, he joined Massey University as a Lecturer at the Institute of Information Sciences and Technology. Currently he is a Senior Lecturer with the School of Engineering and Advanced Technology at Massey University, Palmerston North, New Zealand. His research interests include wireless and mobile communications \& applications, multicarrier, MIMO \& spread spectrum systems, and cooperative communication networks. Dr. Gui is a Senior Member of IEEE and serves as a regular reviewer for a number of quality professional journals and international conferences. He is also a founding member of the Joint Chapter in Communications, Signal Processing and Information Theory established in 2009 under the IEEE New Zealand Central Section.

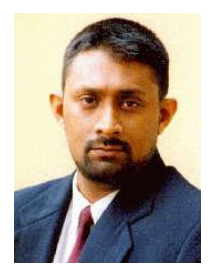

Amal Punchihewa obtained a bachelor of engineering, specializing in Electronics and Telecommunication Engineering, from the University of Moratuwa in Katubedda, Sri Lanka, with honours in 1986. In 1991 he completed a Master of Electronics Engineering at the Technical University of Eindhoven, The Netherlands, focusing on digital video signal processing. Amal has worked as an engineer in both academia and industry over the past twenty-four years, starting as a computer engineer in 1986. After three years he moved to the broadcast industry, working as a research engineer for the national television broadcaster in Sri Lanka. In 1994 he became a senior lecturer at the University of Moratuwa, before migrating to New Zealand in 2002, where he joined Massey University in Palmerston North. His PhD was on framework for rapid evaluation of image and video compression artefacts objectively. He is a senior lecturer and his current research interests are objective assessment of compression artefacts and image processing for surveillance, multimedia communication and care of the elderly. Amal is a fellow of the Institution of Engineering and Technology, a member of Institution of Professional Engineers New Zealand, and a life member of the Sri Lanka Association for Advancement of Science. In 2000 he was awarded the Wimalasurendra award in recognition of the contribution he made to the broadcasting development by the Institution of Engineers Sri Lanka. 